\title{
LINK DEPENDENT ADAPTIVE RADIO SIMULATION
}

\author{
Tara Pun, Deepak Giri \\ Faculty Advisors: \\ Dr. Farzad Moazzami, Dr. Richard Dean, Dr. Arlene Cole-Rhodes \\ Department of Electrical and Computer Engineering \\ Morgan State University
}

\begin{abstract}
This paper shows the optimized Link Dependent Adaptive Radio (LDAR) using the variable QAM OFDM modulation size which adapts to channel conditions. The LDAR enhanced performance is illustrated by use of a flight path simulation with associated channel dynamics. These channel dynamics along the flight path and its effects on the radio link parameters are also discussed.
\end{abstract}

\section{INTRODUCTION}

Radio channel is an integral part of any wireless communication system. Radio channels are unpredictable, and dynamic. Channel conditions affect performance in several ways including noise, multipath, Doppler and other effects. In highly dynamic environments such as aeronautical communication where the speed of aircraft exceeds mach1, channel dynamics, specially fading of the channel becomes even a bigger issue. Furthermore data demand is growing everyday which causes spectrum scarcity for all users in the telemetry community.

Dedicated links between the ground station and the test article has proven to be an inefficient bandwidth utilization method. The integrated network enhanced telemetry (iNET) project - supported by Test Resource Management Center (TRMC) - has aimed at abandoning the point to point link dedication and moving toward networked telemetry. Georgia Tech Research Institute (GTRI) and Morgan State University have undertaken a project within iNET aimed at a bandwidth efficient and adaptive system for aeronautical communication. This work is directed at mitigating the high variance of the aeronautical channels and highly dynamic channel impulse responses of such channels, Link dependent adaptive radio (LDAR) is an effort to maximize the throughput for telemetry links while ensuring an acceptable level of data quality and reliability. This effort includes the development and test of a prototype system that adapts its modulation scheme and error correction coding rate based on channel conditions in a telemetry environment in real time. LDAR selects a modulation scheme that maximizes throughput while ensuring a minimum level of link quality given the current channel conditions. If the quality of channel (SNR or delay spread) is improved, a control signal informs both communication parties to switch to a higher data rate. This increase in data rate is drawn from a table with information on multiple modulation schemes and multiple coding rates. Later on if the error exceeds the predefined acceptable threshold, the same mechanism ensures that next transmission happens in a lower data rate, either by reducing the modulation 
size or by the coding rate [1]. In iNET standard, two types of modulation are considered; SOQPSK and OFDM-QAM [2]. This paper focuses on OFDM-QAM signaling.

The technical approach to this effort is focused on the OFDM modulation scheme which incorporates BPSK, QPSK, 16-QAM, and 256-QAM modulations shown in figure 1, and Convolution coding rates such as, no coding, 3/4 coding and $1 / 2$ coding. This provides 12 options as shown in table 1.

Table 1: Variable rate Transmission choices

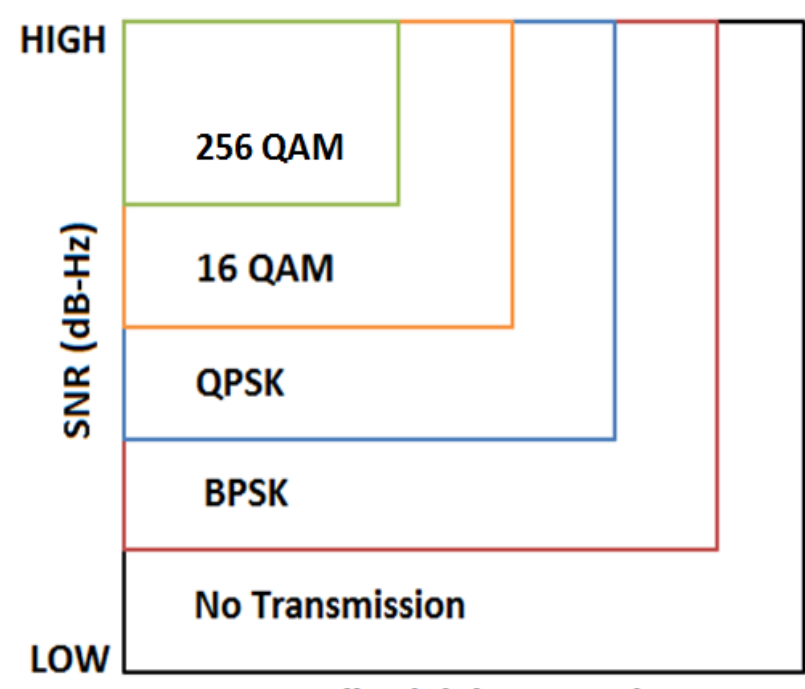

Normalized delay spread

\begin{tabular}{|cccc|}
\hline$\#$ & Modulation & $\begin{array}{c}\text { Code } \\
\text { Rate }\end{array}$ & $\begin{array}{c}\text { Data } \\
\text { Bits/Symbol }\end{array}$ \\
\hline $\mathbf{1}$ & BPSK & No coding & 1 \\
\hline $\mathbf{2}$ & BPSK & $1 / 2$ & 0.5 \\
\hline $\mathbf{3}$ & BPSK & $3 / 4$ & 0.75 \\
\hline $\mathbf{4}$ & QPSK & No coding & 2 \\
\hline $\mathbf{5}$ & QPSK & $1 / 2$ & 1 \\
\hline $\mathbf{6}$ & QPSK & $3 / 4$ & 1.5 \\
\hline $\mathbf{7}$ & 16 QAM & No coding & 4 \\
\hline $\mathbf{8}$ & 16 QAM & $1 / 2$ & 2 \\
\hline $\mathbf{9}$ & 16 QAM & $3 / 4$ & 3 \\
\hline $\mathbf{1 0}$ & 256 QAM & No coding & 8 \\
\hline $\mathbf{1 1}$ & 256 QAM & $1 / 2$ & 4 \\
\hline $\mathbf{1 2}$ & 256 QAM & $3 / 4$ & 6 \\
\hline
\end{tabular}

Figure 1: Link dependent adaptation

OFDM is a combination of modulation and multiplexing. It is a powerful modulation technique that increases bandwidth efficiency and reduces the multipath effect. The occurrence of multipath fades the selected tones carrying data which can be recovered using error correcting codes. OFDM also incorporates a cyclic prefix to remove inter-symbol interference and to maintain orthogonality in the presence of multipath [1].

\section{SYSTEM MODEL}

The primary purpose of every communication system is to deliver the maximum throughput. Data has to be transmitted from transmitter to receiver.

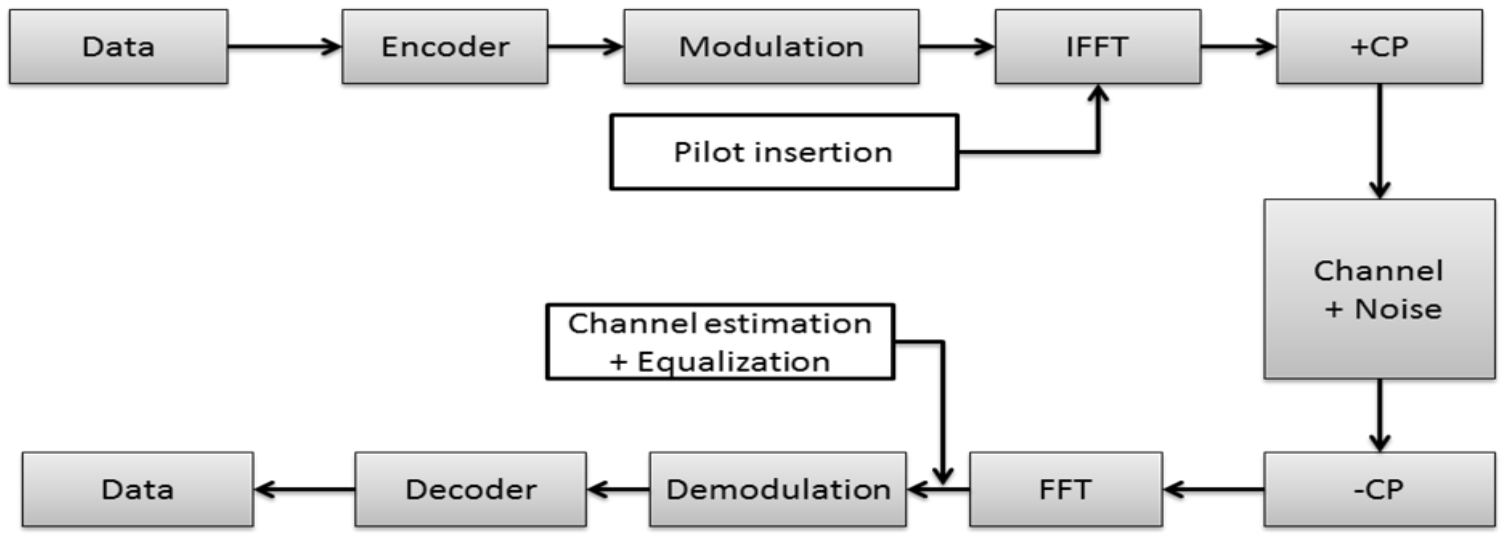

Figure 2: System model 
The data is passed on to encoder where additional code bits are inserted. It decreases the probability of data loss. There are various types of coding available, but for this particular project, the coding has not been included. The encoded data is then passed down for modulation. This project adopts OFDM with BPSK, QPSK, 16-QAM, and 256-QAM modulation schemes. IFFT is applied and pilot bits are inserted. This will help in equalization, synchronization, control, or reference purpose.

The data signal is now transmitted through the channel. Channel effects such as noise, distortion, fading and Doppler result in the low SINR in the signal. At the receiver end, previously added cyclic prefix is removed. Then the signal is transformed into the frequency domain using FFT. In demodulation, the transmitted data is extracted out from the carrier frequency. Channel Estimation is used to predict the original signal sent by the transmitter using pilot bits. Equalization is performed to reverse the distortion occurred while passing through the channel. Then signal is passed into the decoder where the previously added code data are removed. Finally the transmitted data is obtained at the receiver.

In an earlier work [1], variable coding rate OFDM for aeronautical channel were developed and simulated. In this paper a flight scenario has been developed as a test bed for Link Dependent Adaptive Radio simulation. The aeronautical radio telemetry channel is a complex wireless connection between an aircraft and a base station. Such channels are complicated by the multipath distortion and the Doppler related to high speed aircraft [1]. If the channel variations are slow enough, then over a short time interval, the channel can be modeled as linear, time-invariant system whose complex baseband impulse response is of the form:

$$
\tilde{h}(t)=\sum_{k=0}^{L-1} \tilde{\Gamma}_{k} \exp \left\{-j \omega_{c} \tilde{\tau}_{k}\right\} \delta\left(t-\tilde{\tau}_{k}\right)
$$

Where $\tilde{\Gamma}_{k}$ is the complex gain of the $k$-th propagation path, $\tilde{\tau}_{k}$ is the propagation delay of the $k$ th propagation path, and $\omega_{c}$ is the RF carrier frequency [3]. The typical airborne connection can be represented as a 2 ray model with just the direct path and a reflected path. Such a channel has been captured by Dr. Rice [1].

\section{FLIGHT SCENARIOS}

Inspired from LDAR, a flight path demo has been developed which simulates the flight path for test article (TA) and selects the modulation scheme based on the channel condition.

The flight path demo has three different phases: Taxi, Take off, and Cruise as shown in figure 3 below. Rice channels are used in this simulation[4].

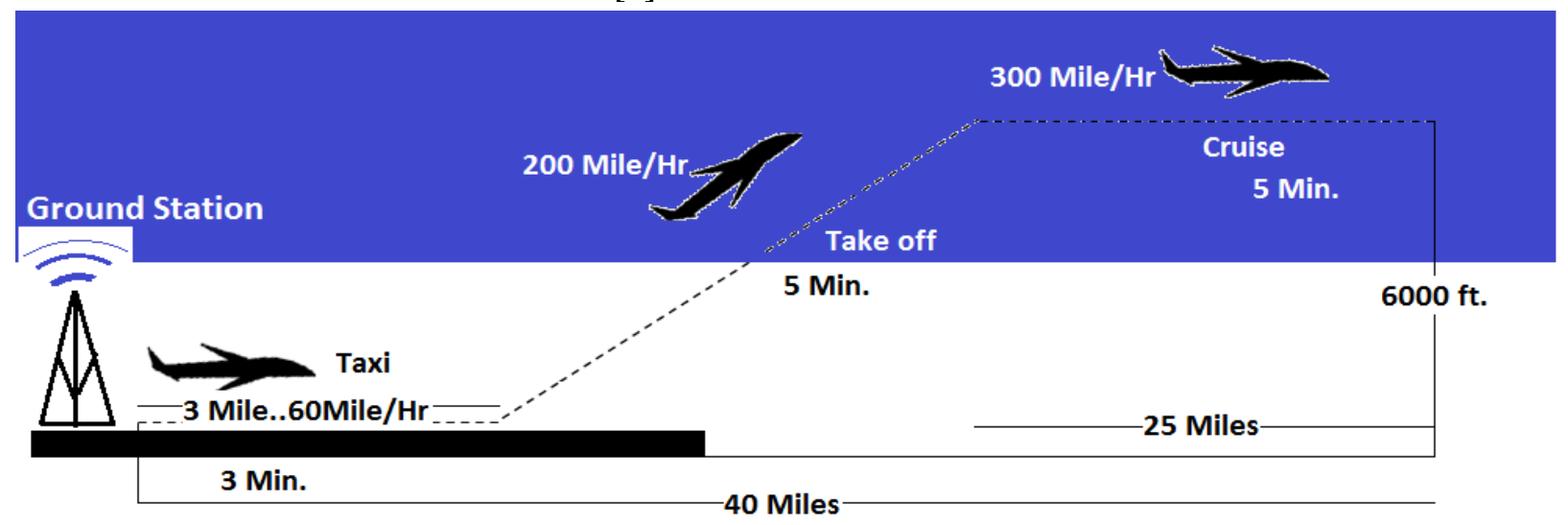

Figure 3: Simulated flight scenario 
The first stage is taxi. This is the phase that TA moves away from the ground station with ground speed of 60 miles per hour for three miles. In the takeoff phase, the aircraft elevates with an angle of 45 degrees with 200 miles per hour speed for 5 minutes. In the cruise phase, the aircraft cruises at the height of 6000 feet with speed of 300 miles per hour for 25 miles. The next phase is to come back through same path to the ground station with same parameters.

\section{Taxi}

In this phase, the aircraft travels 3 miles for takeoff. A cleaned up version of taxi runway channel from Dr. Rice's measurements is chosen for this MATLAB simulation [3]. Figures 4 and 5 show the impulse response and frequency response of this channel respectively.

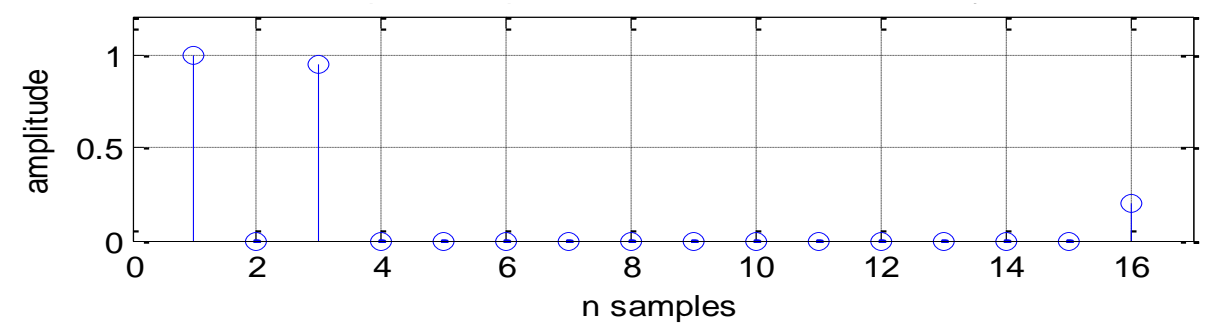

Figure 4: Impulse response of taxi channel

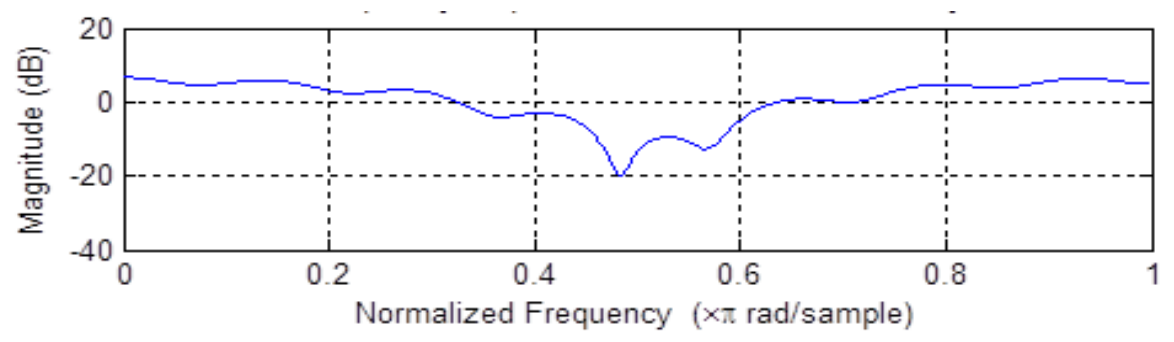

Figure 5: Frequency response of taxi channel

There are three significant taps in this channel. The first and second impulses denote the direct path and ground bounce respectively. The third path with a high delay spread represents the clutter associated with small scale fading. The frequency response of the channel shows two deep nulls, one of which is as low as $-20 \mathrm{~dB}$.

\section{Take off}

Figures 6 and 7 show the impulse response and frequency response of this channel respectively.

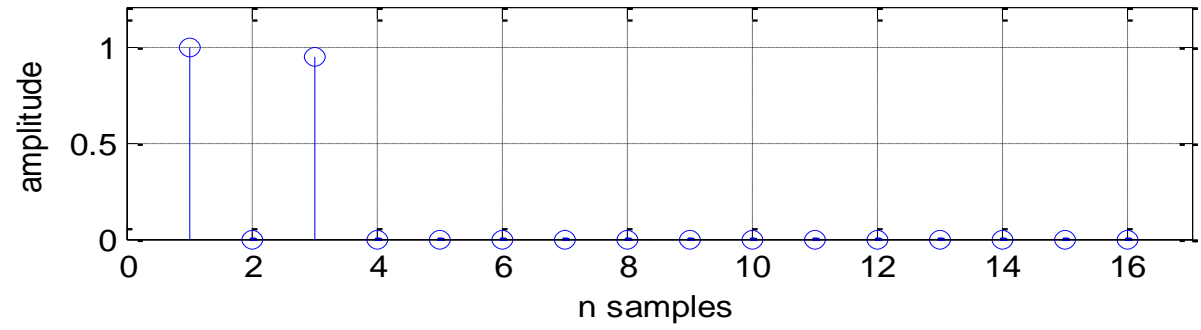

Figure 6: Impulse response of take off channel 


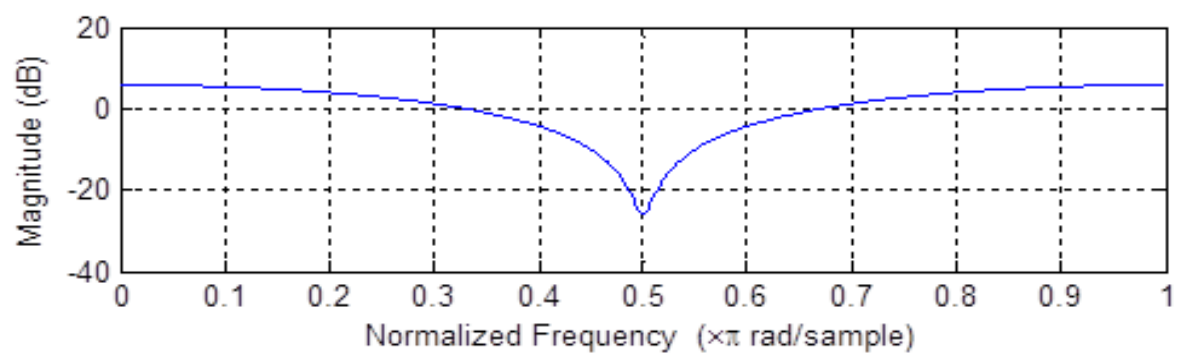

Figure 7: Frequency response of take off channel

In this phase, the TA elevates for approximately 13 miles. This channel is an approximation drawn from a real channel sounding [3]. The first two impulses shown at figure 6 are direct line of sight and reflected path respectively. There are no clutters in this channel. The frequency response at figure 7 shows a deep null below $-20 \mathrm{~dB}$.

\section{Cruise}

In this phase, the aircraft flies away for approximately 25 miles. Figures 8 and 9 show the impulse response and frequency response of this channel respectively.

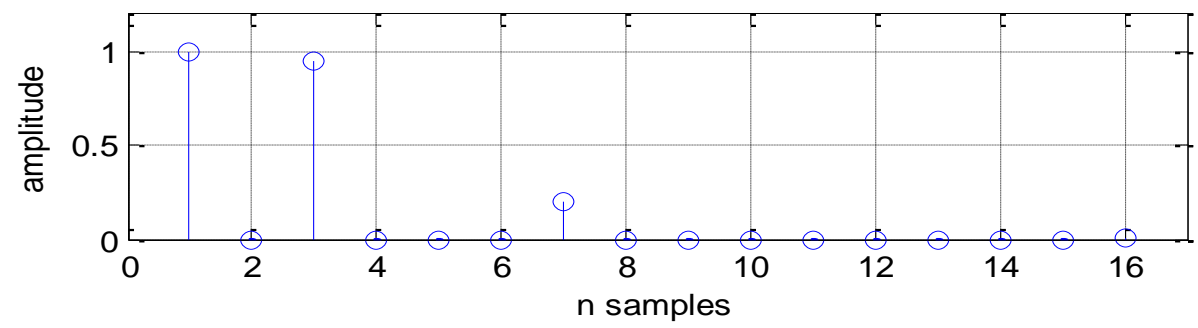

Figure 8: Impulse response of cruise channel

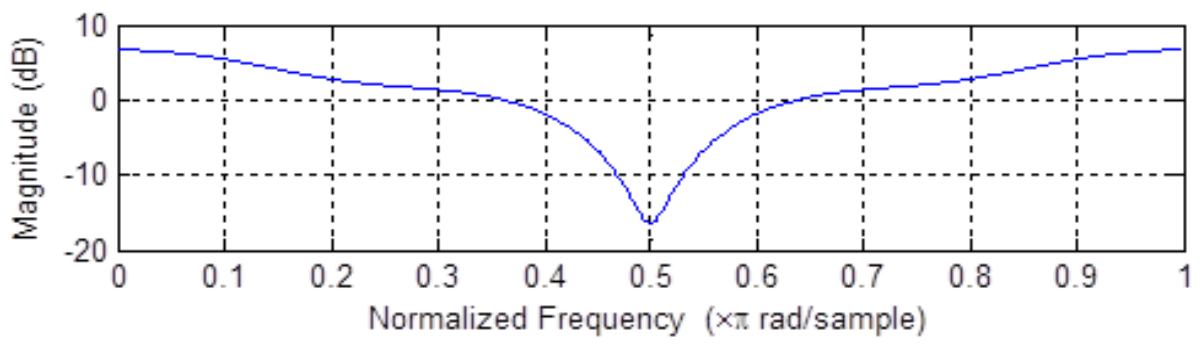

Figure 9: Frequency response of cruise channel

The impulses response of this channel at figure 8 shows the direct line of sight reflected path respectively. The frequency response of this channel at figure 9 shows a deep null below $-15 \mathrm{~dB}$.

\section{SNR PROFILE}

The SNR profile of the flight path is the parameter used in the development of flight demo. This SNR profile provides the signal to noise ratio value for the path being taken by the TA. These SNR values are generated by simulating the path loss using a log distance path loss model with path exponents that vary 
from 2 to 4 as a function of the flight stage. It is done to make the channel more realistic. For this purpose, this flight path demo also uses the channel simulator.

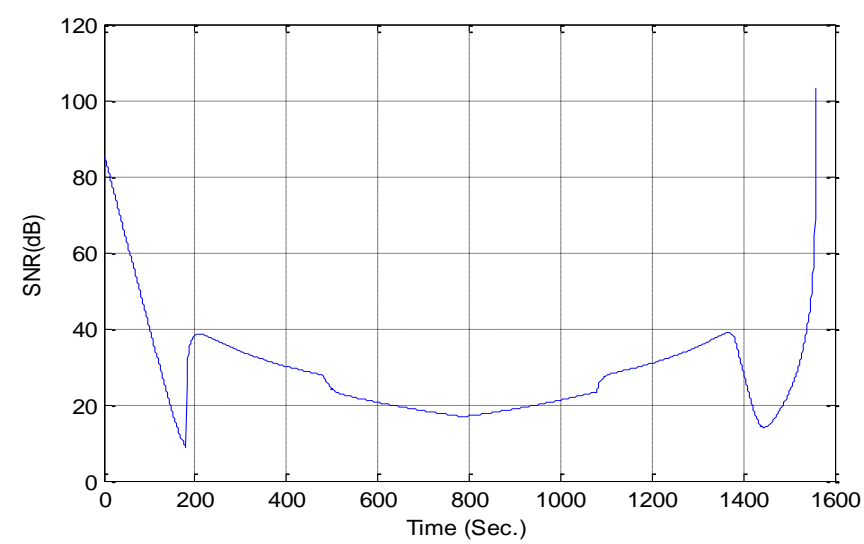

Figure 10: SNR profile of the flight path

Figure 10 shows the SNR values for the complete flight path of the aircraft which takes U-turn at the time 800 seconds. The steep slope of the SNR for first 200 second is used for the Taxi phase of the flight demo. Similarly, SNR values from 200 to 500 second are used for the takeoff phase, and from 600 to 800 seconds for cruise phase. After 800 seconds TA returns to the base using its original course and SNR profile.

\section{QAM SIZE ADAPTATION}

The primary purpose of this project is to select of the QAM modulation size based on the channel conditions. In order to select the best modulation size with the current channel, there has to be a threshold parameter directly related to the channel condition which switches the modulation scheme. In this project, symbol error rate (SER) is used as the threshold parameter which helps to switch the modulation scheme based on the level of error performance. If the performance of the channel fluctuates above and below the threshold, the modulation scheme is adopted accordingly.

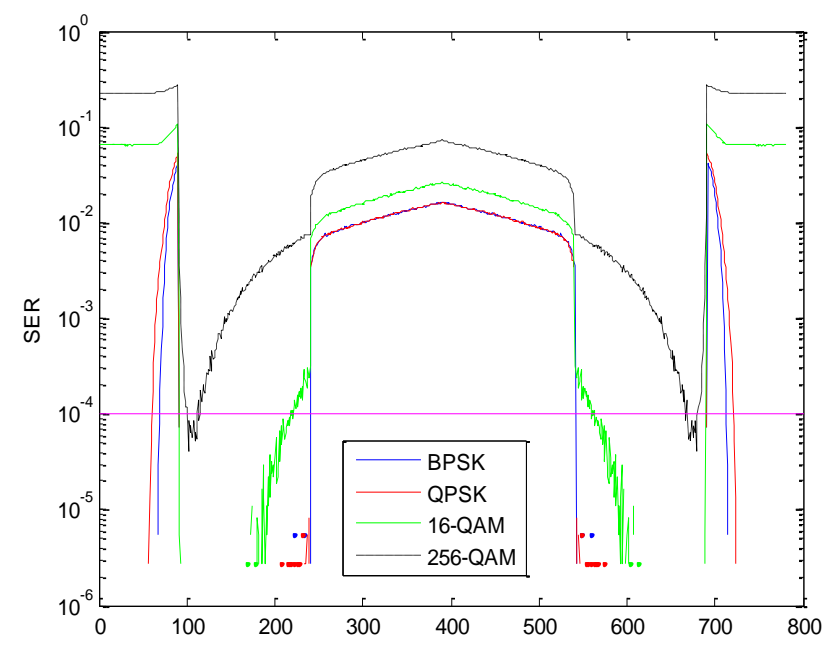

Figure 11: SER values for variable QAM size for flight path without FEC 
Figure 11 shows SER curves for BPSK, QPSK, 16-QAM and 256-QAM. The modulation scheme is selected based on highest available data rate which satisfies the SER threshold. $10^{-4}$ threshold line is shown on figure 11 as an example. The graph represents the selection of high data rate transmission at higher modulation scheme above the threshold value. When the channel has low SNR, it selects 256-

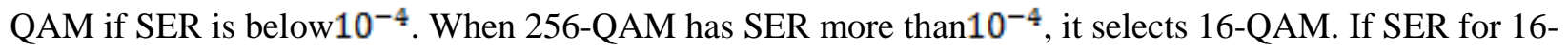
QAM is more than $10^{-4}$, it selects QPSK, and if SER for QPSK is more than $10^{-4}$, it selects BPSK respectively.

Figure 12 shows several snapshots of the flight path simulation scenario taken at different time throughout the flight. Different QAM sizes are autonomously selected for the optimum throughput. The number of transmitted bits is monitored in the simulation and is plotted on the top right corner of the simulation screen. The throughput is increased compared to iNET baseline by several folds.

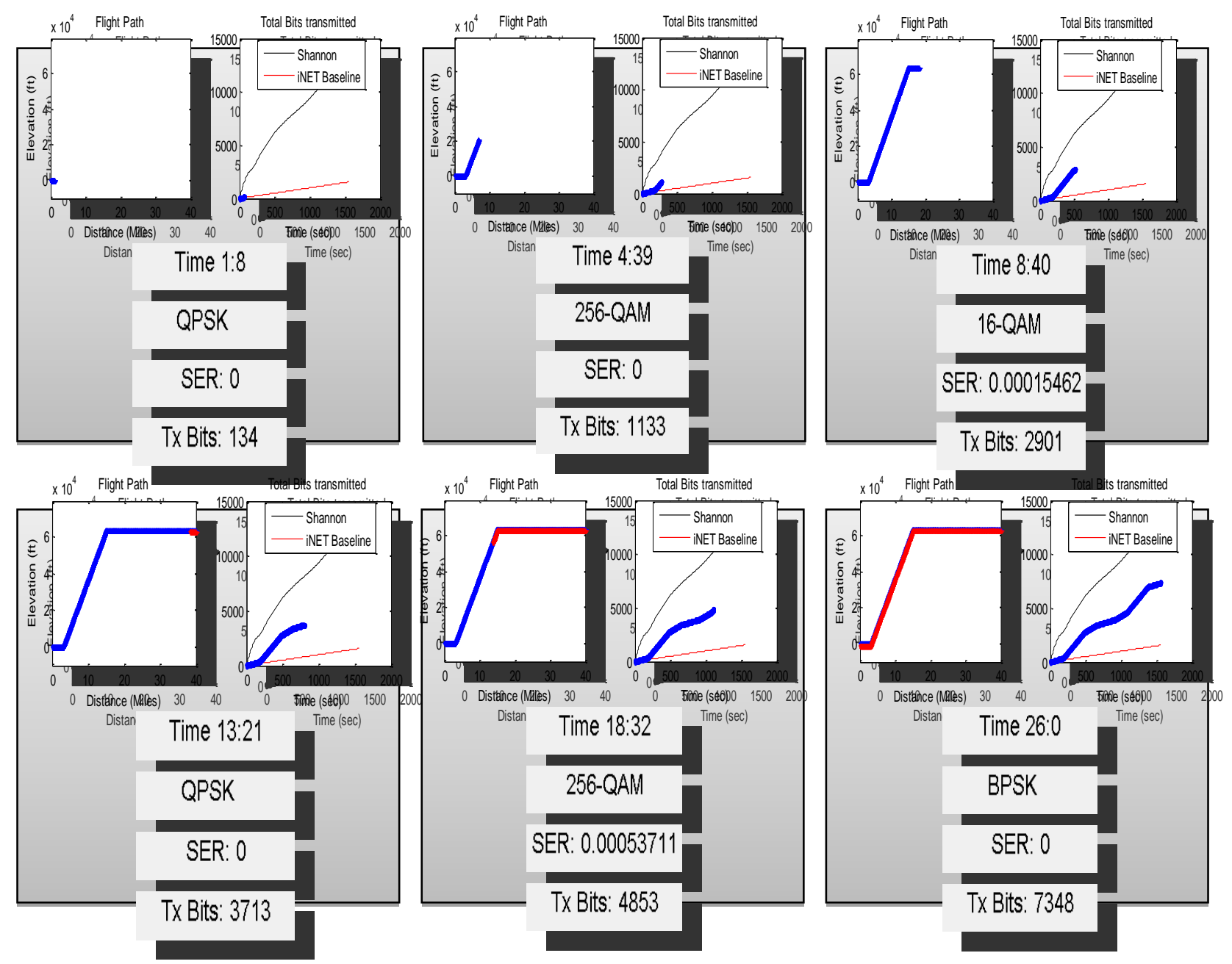

Figure 12: LDAR flight simulator snapshots 


\section{FUTURE WORK}

In addition to the QAM size, the second parameter to be adapted based channel condition is the error correction coding rate within the LDAR project. Figure 13 shows the error performance of the M-QAMOFDM modulation with no coding, rate $1 / 2$ and rate $3 / 4$ coding over AWGN channel. Next step in this work would be the extension of the parameter selection decision table to include coding rate and QAM size together to further improve the throughput. This extension seems reasonable based the separation if the error curves on figure 13 with respect to the coding rates.

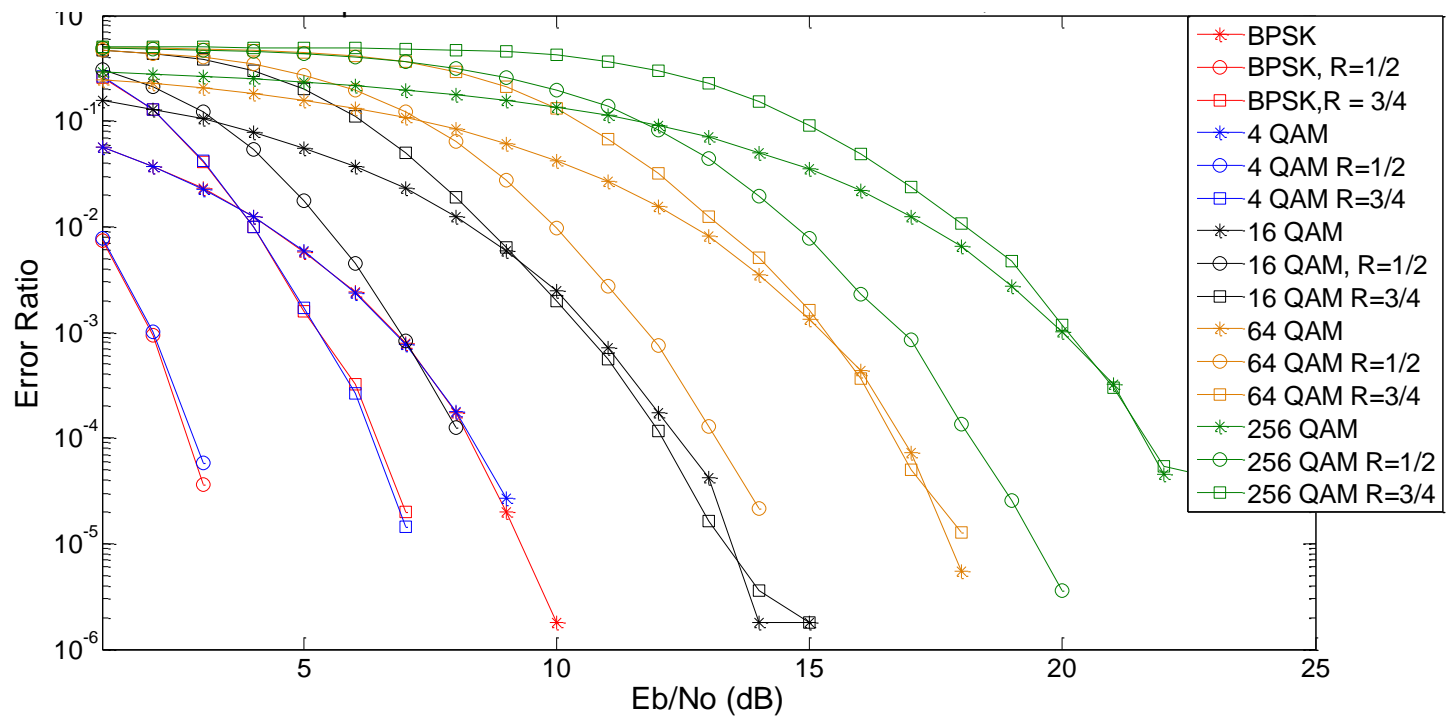

Figure 13: SER curves for M-QAM_OFDM modulation with different coding rates over AWGN

\section{CONCLUSION}

In conclusion, the Link Dependent Adaptive Radio can be a powerful method to optimize iNET data throughput. Since, the channel condition determines the data rate. This adaptive mechanism over the modulation helps minimize the error performance. Also, flight path scenario simulation has shown that the adaptation over the data rate by switching the modulation scheme based on the channel condition provides greater throughput. The result will improve when the coding mechanism is added as part of future work.

\section{ACKNOWLEDGEMENTS}

This work was funded by the Test Resource Management Center (TRMC) Test and Evaluation Science and Technology (T\&E/S\&T) Program under contract W900KK_13-C-0024 (LDAR) and subcontract \#D6949-S1 from Georgia Tech. Research Institute (GTRI). Approved for public release; distribution is unlimited. 412 TW-PA-14317. 


\section{REFERENCES}

[1] M. Elrais, B. Mengiste, B. Gautam and E. Dambia, "Variable OFDM Performance on Aeronautical Channel," in International Telemetry Conference, Las Vegas, 2013.

[2] Physical Layer Modulation, Communication Link Standard for iNET, CTEIP, ver.07, 2010

[3] M. Rice, A. Davis and C. Bettweiser, "Wideband Channel Model for Aeronautical Telemetry," 2004

[4] T. Ha T., Theory and Design of Digital Communication Systems, New York: Cambridge University Press, 2011.

[5] W. Rummler, "A new selective fading model: Application to propagation data," Bell System Techincal Journal, vol. 58, no. 5, pp. 1037-1071, 1979

[6] T. S. Rappaport, Wireless Communications Princples and Practice, Upper Sadde River: Pretince Hall 